\title{
Anterior obturator hip dislocation concurrent with vertical shear fracture of femoral head treated with surgical dislocation of hip-a case report
}

\begin{abstract}
We report a peculiar case of 27-year-old male showing right anterior obturator hip dislocation concurrent with complete femoral head fracture of vertical shear type. Initial radiological studies revealed anteriorly dislocated femoral head with complete fracture of anteromedial femoral head leaving a large bone fragment within hip joint. Despite of closed reduction, radiogram revealed incongruity with fracture surface reversed. We fixed successfully the fracture through Bernese trochanteric flip osteotomy followed by surgical hip dislocation. Anterior obturator dislocation of hip is very rare and often accompanied by impaction fracture of femoral head, which is caused by high energy collision of femoral head with sharp anterolateral margin of obturator foramen with hip flexed and externally rotated. If strong enough to break bone, this impact will make vertical shear fracture of femoral head similar to our case. Because there is no written report of this injury treated with surgical hip dislocation with plausible mechanism of injury, we report this case.
\end{abstract}

Volume I Issue 3 - 2014

\author{
Suhyun Cho \\ Department of Orthopaedic Surgery, Korea
}

Correspondence: Suhyun Cho, Department of Orthopaedic Surgery, Good Samsun Hospital, 326 Gaya daero, Sasang gu, Busan 617-718, Korea, Tel 82513109289,

Email SuhyunII23@knaver.com

Received: October 24, 2014 | Published: November 06, 2014

Keywords: Obturator hip dislocation, Femoral head fracture, Surgical hip dislocation

Abbreviations: MFCA, Medial Femoral Circumflex Artery; OHD, Obturator Hip Dislocation; SHD, Surgical Hip Dislocation

\section{Introduction}

While obturator hip dislocation (OHD) is rarely encountered, it would be much rarer to observe this concurrent with femoral head fracture. We described a peculiar case of OHD concurrent with head fracture which was successfully treated by Bernese trochanteric flip osteotmy followed by surgical hip dislocation (SHD). This SHD is a technique with which full access to the femoral head and acetabulum can be made without the risk of avascular necrosis. ${ }^{1}$ This approach can be used as approach of various intraarticular disease of hip. To our knowledge, because there is no written report of obturator hip dislocation concurrent with head fracture treated with SHD with plausible mechanism of injury, so we report this case.

\section{Case}

A 27-year-old man was injured by automobile accident and immediately presented to our emergency department. On physical examination, right hip was in flexion, external rotation and abduction. He complained of severe pain, and passive and active movements were not possible in right hip. There was neither neurovascular deficiency nor associated injuries. Radiograph of both hip revealed obturator dislocation of right femoral head and fractured femoral head fragment within hip joint (Figure 1). The dislocation was immediately reduced by traction in line of deformity followed by gentle adduction and internal rotation with pelvis stabilized by an assistant. After closed reduction, anteroposterior radiograph showed femoral head was reduced but crescentic radio-opacity was visible overlying femoral head and translateral radiograph revealed that the fractured femoral head fragment was turned inside out during closed reduction (Figure $2 \mathrm{~A} \& 2 \mathrm{~B})$. Computed tomogram depicted the 3-D configuration of fracture (Figure 2C \& 2D)

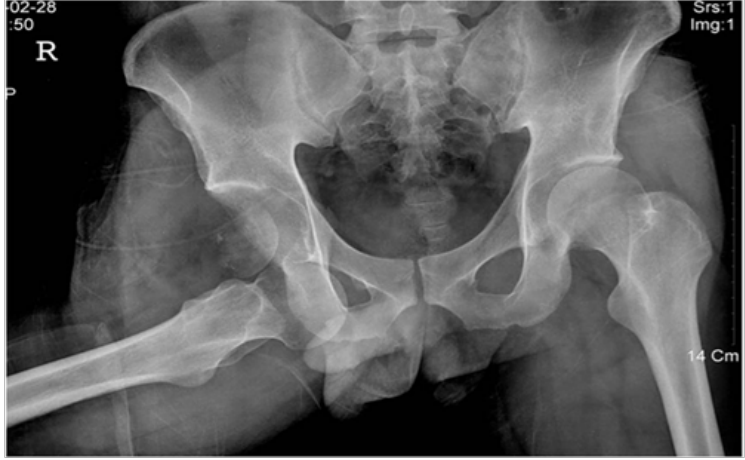

Figure I Right lower extremity is flexed, abducted and rotated externally while the femoral head is dislocated anteriorly with fractured head fragment within the confine of acetabulum.

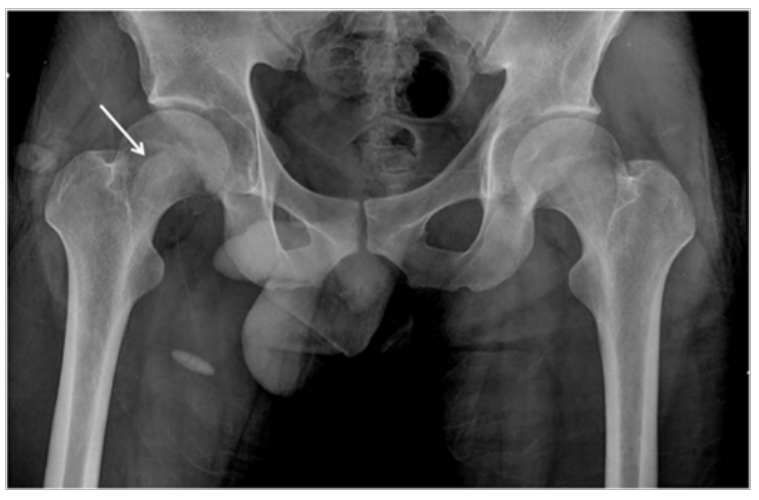

Figure 2a Both hip anteroposterior radiogram after closed reduction showing reduced femoral head with crescentic radio-opacity (arrow) overlying femoral head still visible. 


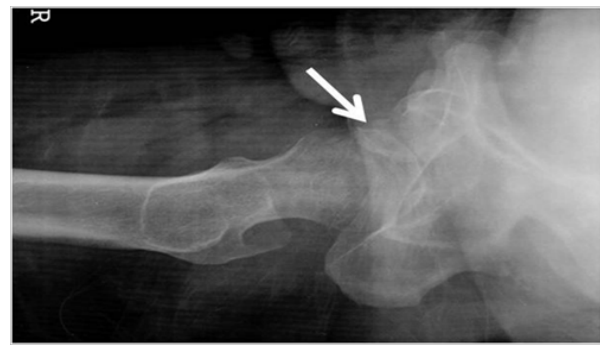

Figure $\mathbf{2 b}$ Hip lateral radiogram revealing that the fractured femoral head fragment was turned inside out (arrow) during trial of closed reduction.

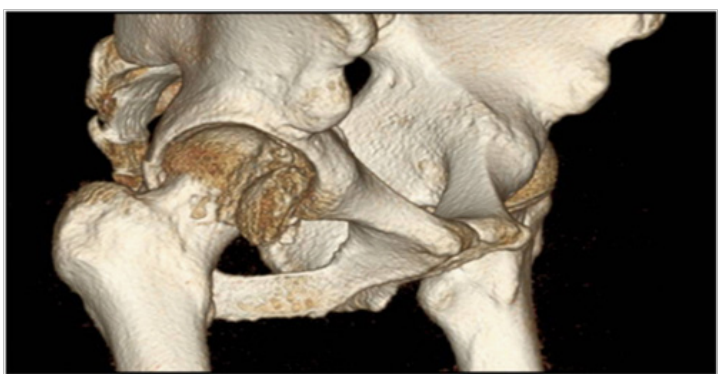

Figure 2c 3-D computed tomogram showing geometry of fracture.

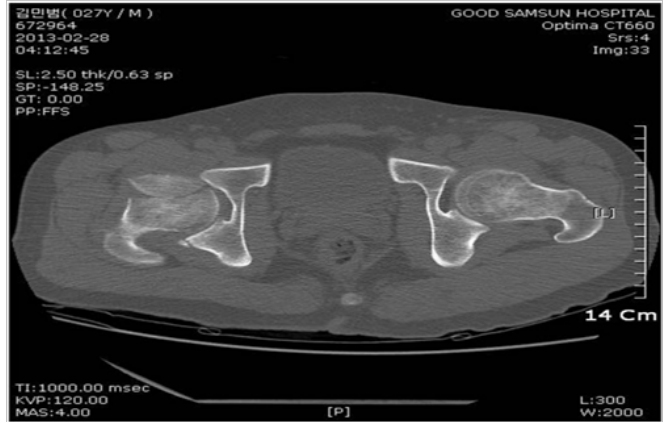

Figure 2d 3-D computed tomogram showing posteroinferior acetabular rim fracture.

We decided to perform open reduction and internal fixation immediately. To preserve posterior vascularity of femoral head, we approached hip joint anteriorly after careful flip osteotomy of greater trochanter. As retracting osteotomized greater trochanter, we were able to find fractured femoral head and its large fragment (Figure $3 \mathrm{~A})$. Fractured fragment was measured about $45 \mathrm{~mm}$ in diameter and was a large part of anteromedial femoral head involving half of ligamentum teres (Figure 3B \& 3C). It was possible to perform accurate reduction and rigid fixation of fractured fragment with two Acutrak screws $(40 \mathrm{~mm}$ and $35 \mathrm{~mm}$, Acumed $\mathbb{R}$, USA) and careful reduction of dislocated hip was performed (Figure 4A \& 4B). Finally we reattached and fixed the osteotomized greater trochanter with three screws. Postoperative simple radiogram and computerized tomogram showed well fixed fracture fragment with mild residual indentation on anterosuperior femoral head (Figure 5A-5C). On postoperative one day, he could ambulate with wheelchair. One week later, he was permitted to walk with crutch while bearing partial weight on his foot. On postoperative one year follow-up, radiogram showed bony union of fractured femoral head as well as osteotomized greater trochanter and healing of fractured posteroinferior acetabular rim without any evidence of secondary osteonecrosis (Figure 6A-6C).

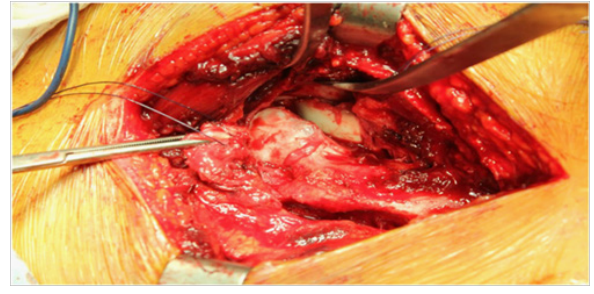

Figure 3a Intraoperative photogram showing reversed large fragment

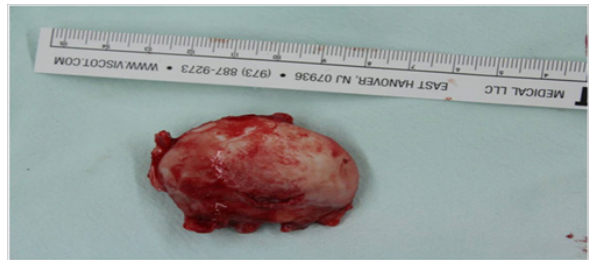

Figure 3b Fractured fragment was measured about $45 \mathrm{~mm}$ in diameter

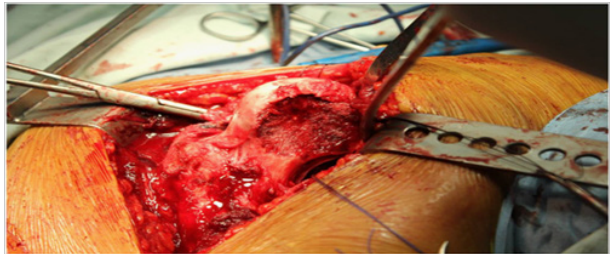

Figure 3c Fracture fragment was a large part of anteromedial femoral head involving half of ligamentum teres.

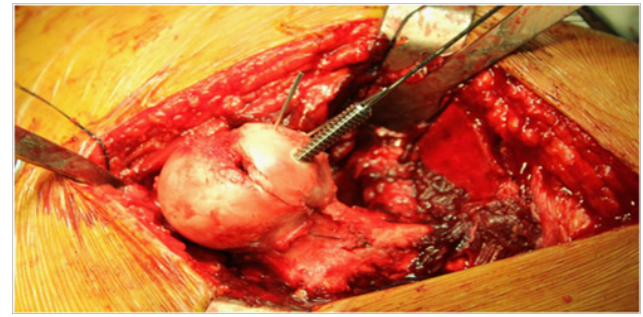

Figure 4a Intraoperative lateral photogram.

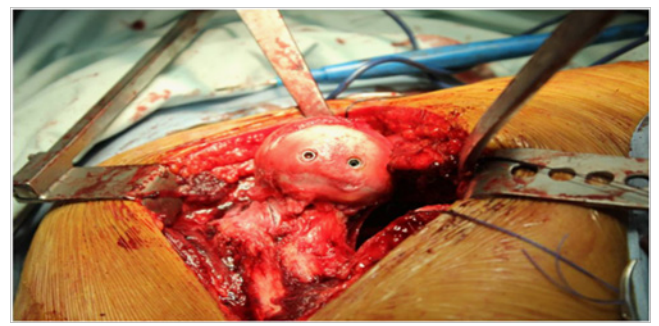

Figure $\mathbf{4 b}$ Intraoperative front photogram showing accurate reduction and rigid fixation of fracture fragment with two Acutrak screws.

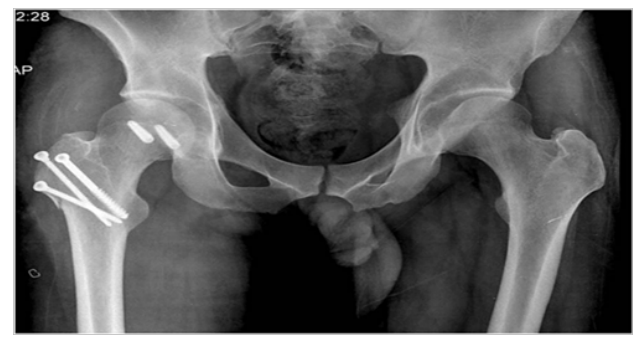

Figure 5a Postoperative both hip anteroposterior radiogram. 


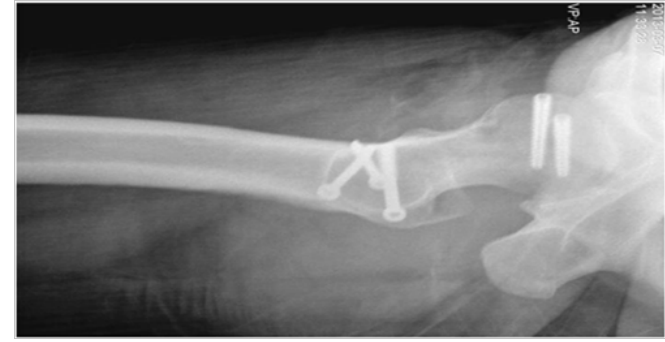

Figure 5bPostoperative lateral radiogram showing well fixed fracture fragment.

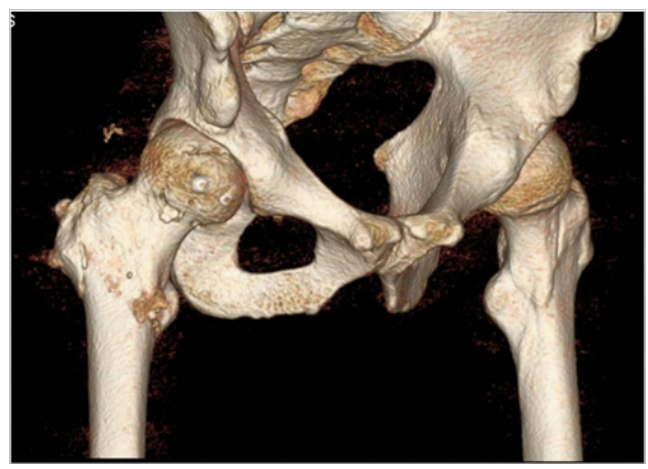

Figure 5c 3-D computed tomogram reveals mild indentation on anterosuperior femoral head.

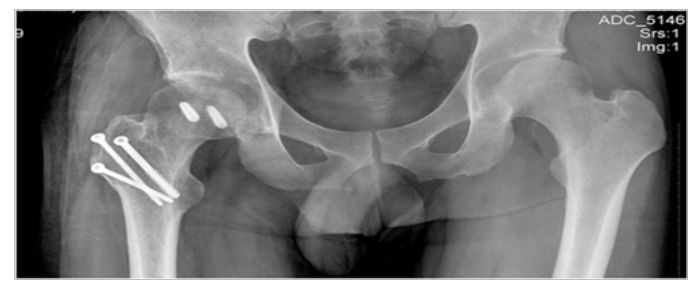

Figure 6a Postoperative one year both hip anteroposterior radiogram.

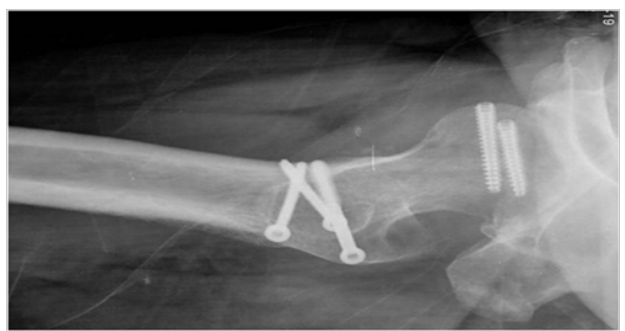

Figure 6b Postoperative one year lateral radiogram showing bony union without any evidence of secondary osteonecrosis.

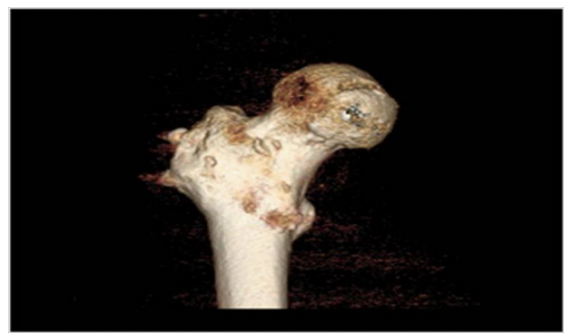

Figure 6c 3-D tomogram reveals mild bone resorption around fracture line on the femoral head.

\section{Discussion}

Anterior dislocation of hip is less common than posterior one, comprising only $9-12 \%$ of all hip dislocations and has been further classified as pubic(superior), obturator(inferior) and perineal type. ${ }^{2}$ Anterior dislocation of hip occurs by abduction and external rotation of the hip. Pubic type is caused by extension, whereas obturator type is caused by flexion as additional momentum. At that second, femoral head rests against sharp anterolateral margin of obturator foramen, causing indentation or impaction fracture on the anterosuperior aspects of femoral head. This fractures were usually reported to be located on the posterosuperior and lateral portions of the femoral head. ${ }^{3,4}$ But, our case was complete fracture of anterior femoral head and the direction of fracture was different from those of previously reported indentation fractures which were from posterolateral to anteromedial. It was on the oblique coronal plane, from anterolateral to posteromedial (Figure 4). Yoon et al. ${ }^{5}$ reported indentation fracture of femoral head of similar direction, which occurred without hip dislocation and was asserted to be caused by low energy injury in patient with general laxity. But we think the direction of fracture line may be just related to how much the hip is flexed (Figure 7A \& 7B).

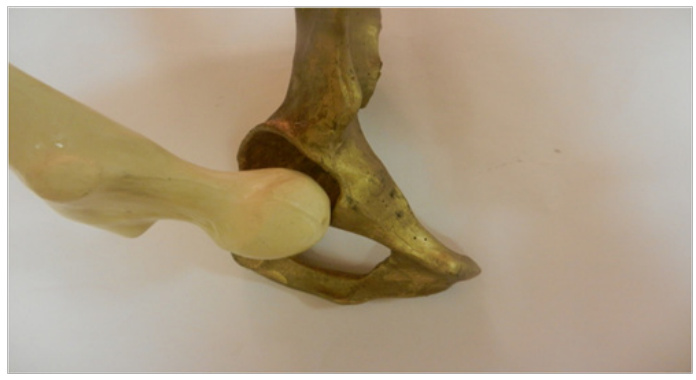

Figure 7a Direction of fracture line is from anterolateral to posteromedial on femoral head with hip fully flexed.

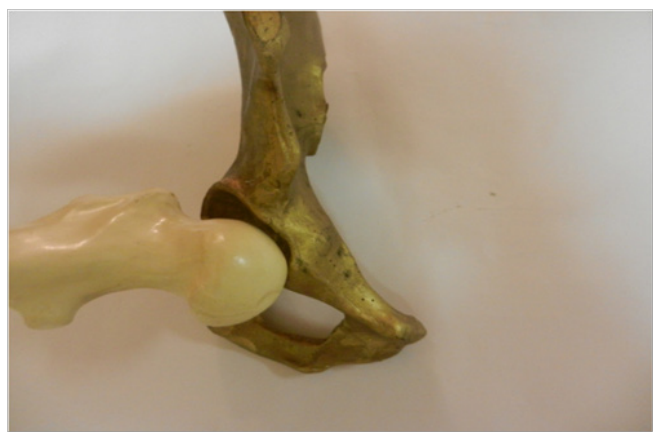

Figure 7b From anteromedial to posterolateral on femoral head with hip moderately flexed.

Vertical shearing could be conjectured as initiating force for fracture based upon indentation and cartilage defect of anterosuperior femoral head (Figure 4A). The possible mechanism of this injury is as follows. At the moment of anterior dislocation in highly flexed position, anterosuperior femoral head became to collide with anterior edge of acetabulum. At this point, medially directed force was abruptly exerted to cause vertical shear fracture of anterosuperior aspect of femoral head (Figure 8A-8C). Van der WerKen et al. ${ }^{6}$ also proposed that a fracture of the femoral head may precede its dislocation and this fracture is caused by direct blow upon greater trochanter while forces are resisted by firm superior portion of the acetabulum. To cause complete shear fracture, the direction of force 
should accurately be toward anterior edge of acetabular rim, and the amount and velocity of impact ought to be adequate enough. That is why majority of femoral head fracture reported were just indentation fracture. Rim fracture of posteroinferior acetabulum was caused by abrupt collision of posterosuperior femoral neck with posteroinferior margin of acetabulum on the edge of being dislocated anteriorly. Of OHDs, this case accompanied by complete femoral head fracture is assumed to be caused by high energy injury and advanced form of either indentation fracture of Brumback type $4 \mathrm{~A}$ or tranchondral shear of Brumback type 4B.?

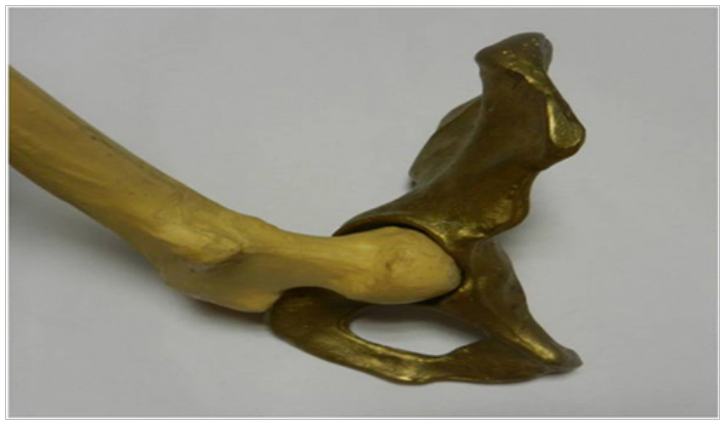

Figure 8a Firstly, femoral head still within confine of acetabulum with hip abducted, flexed and externally rotated.

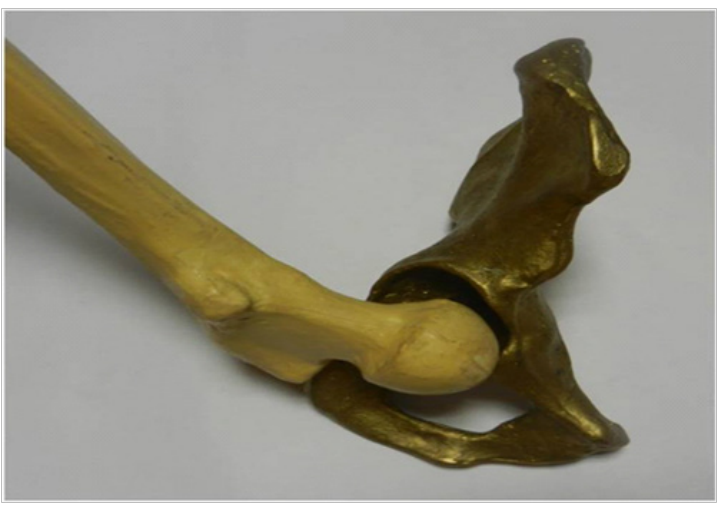

Figure $\mathbf{8 b}$ Secondly, femoral head is about to come out of acetabulum while abutting its anterior edge. At this stage, strong external force exerted upon greater trochanter may cause vertical shear fracture of femoral head.

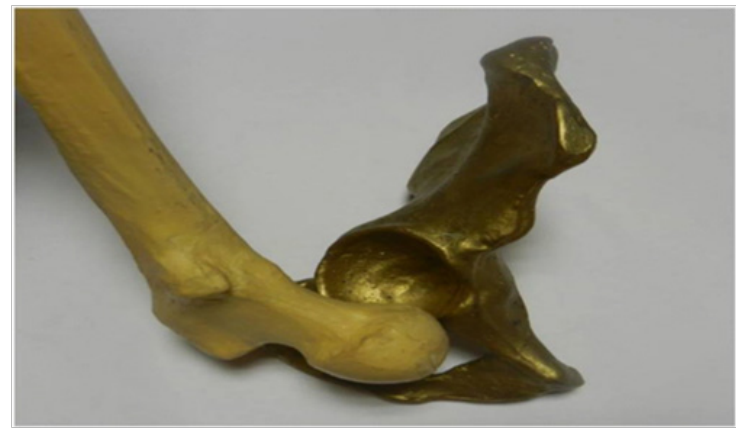

Figure 8c Thirdly, fractured femoral head is dislocated completely with fracture fragment remained within confine of acetabulum.

When choosing the direction of surgical approach in managing hip dislocation, we'd better choose the side of dislocation, for approaching through the side of dislocation may prevent secondary avascular necrosis of femoral head by obviating the possibility of violation of the other side vascularity. In addition, it was not only difficult to visualize the anterior head fragment through the posterior Kocher-Langenbeck approach, but adequate reduction and fixation of femoral head was unpredictable of anterior approaches; SmithPeterson approach does provide direct access to the anterior surface of femoral head and might be feasible only for excision of comminuted fragments and debris. ${ }^{8}$ Furthermore, this approach might not allow us to address posteroinferior acetabular rim fracture shown in this case. SHD advocated by Ganz et al. ${ }^{1,9}$ enabled us to inspect entire femoral head and full circumference of acetabulum. Furthermore it was possible for us to fix the fractured anterior femoral head accurately with the interface between fracture fragment and the remainder of the femoral head compressed rigidly as we could access the entire femoral head outside acetabulum (Figure 4). Headless compression screw used in this case may eliminate the need to countersink head, so is very effective means of interfragmentary compression in treatment of fracture of femoral head. Bastian et al. reported that SHD with a trochanteric flip osteotomy is a simple, one-step technique that allows full inspection of the hip to treat osteochondral femoral defects occurred in OHD by osteochondral transplantation. ${ }^{2}$ In treating an OHD with concomitant femoral head fracture caused by vertical shear, we also approached hip joint through SHD with a trochanteric flip osteotomy not compromising posterior medial femoral circumflex artery (MFCA) and were able to fix the fractured fragment rigidly. Our main concern was development of avascular necrosis at longer follow-up although there was no evidence of this at one year followup. Avascular necrosis may be attributable to compromise of MFCA either at the moment of dislocation or during surgical approach. Trochanteric osteotomy and SHD may lead to avascular necrosis of femoral head. When performing this trochanteric flip osteotomy, it is very important to preserve MFCA. We developed the interval between piriformis and gluteus minimus muscle just leaving a shell of posterosuperior trochanteric overhang with attached gluteus muscle in order to protect MFCA. When depicting osteotomy line on posterior trochanter, we kept proximal osteotomy plane superficial to piriformis to protect MFCA which is beneath piriformis. The MFCA of this case was thought to be intact at the moment of anterior dislocation because posterior soft tissue remained intact, so it was reasonable to approach hip anteriorly after trochanteric flip osteotomy. Likewise, this SHD in itself was a safe and versatile approach with enough rationale. In 2 large series of SHD, there were no reported cases of avascular necrosis ${ }^{1,10}$ Our second concern was progression of arthritic change following head fracture at longer follow-up although the fracture is away from main weight bearing area of femoral head.

\section{Conclusion}

We report a case of anterior obturator dislocation of femoral head with fractured femoral head caused by shearing force, which appeared to be extreme form of simple obturator dislocation and could be easily approached and managed through Bernese surgical hip dislocation

\section{Acknowledgments}

None.

\section{Conflicts of interest}

The author declares that there is no conflict of interes. 


\section{References}

1. Ganz R, Gill TJ, Gautier E, et al. Surgical dislocation of the adult hip: technique with full access to the femoral head and acetabulum without the risk of avascular necrosis. J Bone Joint Surg Br. 2011;83(8):1119-1124.

2. Bastian JD, Büchler L, Meyer DC, et al. Surgical hip dislocation for osteochondral transplantation as a salvage procedure for a femoral head impaction fracture. J Orthop Trauma. 2010;24(12):e113-e118.

3. Olson SA, Matta JM. Fractures of the acetabulum, hip dislocation and femoral head fracture. In: Chapman MW (Ed.) Chapman's orthopaedic surgery $\left(3^{\text {rd }}\right.$ ed). Lipponcott Williams \& Wilkins, Philadelphia, USA, pp. $587-616$.

4. DeLee JC, Evans JA, Thomas J. Anterior dislocation of the hip and associated femoral-head fracture. J Bone Joint Surg Am. 1980;62(6):960964.

5. Yoon PW, Jeong HS, Yoo JJ, et al. Femoral head fracture without dislocation by low-energy trauma in a young adult. Clin Orthop Surg. 2011;3(4):336-341.
6. Van der Werken C, Blankensteijin JD. Fracture of the femoral head without dislocation: a case report. Acta Orthorp Scand. 1987;58(2):173-174.

7. Brumback RJ, Kenzora JE, Levitt LE, et al. Fractures of the femoral head. Hip. 1987;181-206.

8. Gardner MJ, Suk M, Pearle A, et al. Surgical dislocation of the hip for fractures of the femoral head. J Orthop Trauma. 2005;19(5):334-342.

9. Siebenrock KA, Gautier E, Woo AK, et al. Surgical dislocation of femoral head for joint debridement and accurate reduction of fractures of the acetabulum. J Orthop Trauma. 2002;16(8):543-552.

10. Dussault RG, Beauregard G, Fauteaux P, et al. Femoral head defect following anterior hip dislocation. Radiology. 1980;135(3): 627-629.

11. Sink E, Beaule PE, Sucato D, et al. Multicenter study of complications following surgical dislocation of the hip. J Bone Joint Surg Am. 2011;93(12):1132-1136. 